\title{
Evaluation of the real-time fluorescence loop-mediated isothermal amplification assay for the detection of Ureaplasma urealyticum
}

\author{
Jie-Ni Shen ${ }^{1,2+}{ }^{+}$, Jing-Yi Ye ${ }^{2 \dagger}$, Meng-Xiao Lao ${ }^{3 \dagger}$, Chu-Qiao Wang ${ }^{2}$, Dong-Hong Wu ${ }^{2}$, Xiao-Ying Chen ${ }^{2}$, \\ Li-Hong Lin ${ }^{4^{*}}$, Wen-Yan Geng ${ }^{1,5^{*}}$ and Xu-Guang Guo ${ }^{1,6,7^{*}}$ (D)
}

\begin{abstract}
Ureaplasma urealyticum (UU) is commonly present in human reproductive tract, which frequently leads to genital tract infection. Hence, there is an urgent need to develop a rapid detection method for UU. In our study, a real-time fluorescence loop-mediated isothermal amplification (LAMP) assay was developed and evaluated for the detection of UU. Two primers were specifically designed based on the highly conserved regions of ureaseB genes. The reaction was carried out for $60 \mathrm{~min}$ in a constant temperature system using Bst DNA polymerase, and the process was monitored by real-time fluorescence signal, while polymerase chain reaction (PCR) was performed simultaneously. In real-time fluorescence LAMP reaction system, positive result was only obtained for UU among 9 bacterial strains, with detection sensitivity of $42 \mathrm{pg} / \mathrm{\mu L}\left(4.2 \times 10^{5} \mathrm{CFU} / \mathrm{mL}\right)$, and all 16 clinical samples of $U U$ could be detected. In conclusion, realtime fluorescence LAMP is a simple, sensitive, specific and effective method compared with conventional PCR, which shows great promise in the rapid detection of UU.
\end{abstract}

Keywords: Ureaplasma urealyticum, Loop-mediated isothermal amplification, Real-time

\section{Introduction}

Reproductive tract infection caused by Mycoplasma hominis (MH), Mycoplasma genitalium, and Ureaplasma urealyticum (UU), imposes a threat to human reproductive tract health. Among these, UU is the most prevalent pathogen that colonizes the human reproductive tract (Esen et al. 2017). Additionally, UU causes severe problems for infants and their mothers. As an example,

\footnotetext{
*Correspondence: 81743150@qq.com; gengwenyan0305@163.com; gysygxg@gmail.com

${ }^{\dagger}$ Jie-Ni Shen, Jing-Yi Ye and Meng-Xiao Lao contributed equally to this work.

1 Department of Clinical Laboratory Medicine, The Third Affiliated Hospital of Guangzhou Medical University, Guangzhou, China

${ }^{4}$ Center for Reproduction Medicine, Key Laboratory for Major Obstetric

Diseases of Guangdong Province, The Third Clinical School of Guangzhou Medical University, Guangzhou, China

${ }^{5}$ Department of Blood Transfusion, The Third Affiliated Hospital

of Guangzhou Medical University, Guangzhou, China

Full list of author information is available at the end of the article
}

research has proved that UU could be vertically transmitted from mother to child, thereby increasing morbidity and mortality of newborns (Kafetzis et al. 2004; Sobouti et al. 2014). Moreover, people who have a mixed infection of UU and $\mathrm{MH}$ are prone to cause premature delivery (Kwak et al. 2014; Lee et al. 2016).

Conventional diagnosis of UU infection relies on positive culture, antigen detection, serology, and molecular biology. Generally, polymerase chain reaction (PCR) is one of the most common techniques (Liu et al. 2017). However, the above-mentioned methods require either long culture periods or precision instruments, thus limiting their application in primary medical institutions, especially in developing countries. Apart from that, PCR increases the risk of sample cross-contamination (Dehghan Esmatabadi et al. 2015). Hence, an alternative diagnostic is needed to diagnose UU infection fast and efficiently. 
Loop-mediated isothermal amplification (LAMP), which was first described by Notomi in 2000 (2000), is a promising alternative to conventional PCR techniques. It requires at least 4 specific primers to identify 4-6 distinct sequences of DNA template, which can be less influenced by inhibitory molecules in clinical samples (Enomoto et al. 2005). Due to the characteristics of primers, the concentration of LAMP is higher than that in PCR reaction. The temperature of real-time fluorescence LAMP reaction ranged between 60 and $65^{\circ} \mathrm{C}$, and it merely takes Bst DNA polymerase 30-60 min to amplify target DNA sequence (Ruang-Areerate et al. 2021). Compared with conventional PCR, real-time LAMP increases the reaction rate two to three times (Guo et al. 2018).

The LAMP-amplified products can be observed directly by reaction tubes, and thus avoiding the requirement of thermal cycling and electrophoresis, which enable bedside of Ureaplasma spp. (Fuwa et al. 2018). Hence, with addition of fluorescent dye, real-time fluorescence LAMP shows tremendous ability of quick quantitative detection of UU infection. Though LAMP technology has been utilized for UU detection in clinical research (Fuwa et al. 2018), no research has focused on the establishment and evaluation of UU detection based on real-time fluorescence LAMP. Our study aims to evaluate real-time fluorescence LAMP assay with a modification, which combined with high sensitivity, specificity and efficiency, to investigate its feasibility in clinical practice and facilitate the rapid detection of UU in clinical samples.

\section{Methods}

The study was approved by the institutional ethics committee of Third Affiliated Hospital of Guangzhou Medical University. All patients provided written consent before sample collection.

\section{Bacterial strains and bacterial genomic DNA extraction}

The strains were collected from the Department of microbiology laboratory, the Third Affiliated Hospital of Guangzhou Medical University, where the strains were isolated, identified by VITEK 2 automatic microorganism identification instrument, and stored at $-70{ }^{\circ} \mathrm{C}$. The sources of these genomes are shown in Table 1. The strains used in the present study include Ureaplasma urealyticum ATCC27618, Escherichia coli, Proteus mirabilis, Klebsiella pneumoniae, Enterococcus aerogenes, Staphylococcus haemolyticus, Pseudomonas aeruginosa, Streptococcus agalactiae and Mycoplasma hominis. The protocol was performed as follows. Bacteria suspensions $(1 \mathrm{~mL})$ were prepared from the bacteria colonies in a liquid medium washed by phosphate buffer saline.
Table 1 Bacterial strains used in this study

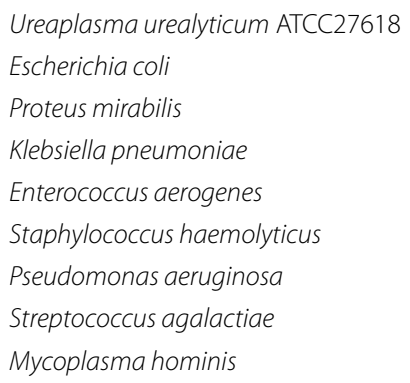

The source of all bacterial strains was: Third Affiliated Hospital of Guangzhou Medical University

The extraction of bacteria DNA was conducted under instruction manuals. Finally, concentration and purity of the extracted specimens were tested on a DNA concentration tester.

\section{Primer design and real-LAMP system}

The primers were designed by the real-time fluorescence LAMP primer design software Primer Explorer V4 based on DNA sequence of UU ureaseB gene (GenBank accession no. AF085726.2). The sequences of primers are listed in Table 2. Primers included forward outer primer (F3), backward outer primer (B3), forward internal primer (FIP), backward internal primer (BIP), loop forward primer (LF), and loop backward primer (LB), which were synthesized by Invitrogen (Shanghai, China). Bacterial genomic DNA extraction kit was purchased from Tiangen Biochemical Technology Company Limited.

The LAMP reaction system was established according to the manual of DNA amplification kit. The LAMP mix without DNA templates was first prepared on ice (Table 3). Two microliters of DNA template were added to $23 \mu \mathrm{L}$ of LAMP mix, to a total volume of $25 \mu \mathrm{L}$. Sterile distilled water was used as a negative control in all tests. The fluorescence channel was selected for TIANGEN kits (Tiangen; Beijing, China) detection. The mix reaction was incubated in a real-time quantitative PCR analyzer (ABI 7500) at $63{ }^{\circ} \mathrm{C}$ for $60 \mathrm{~min}$, then $80^{\circ} \mathrm{C}$ for $2 \mathrm{~min}$ to terminate the reaction. Fluorescent dye of SYTO-9 (Guangzhou Deaou Biotechnology) was added to the tubes. The reaction was considered positive if green fluorescence was observed.

\section{Primer screening experiment and dissociation curve analysis}

Two selected primers, UreaseB-1 and UreaseB-2, were prepared for working solutions. The sampling along with 
Table 2 Primers used for the real-time fluorescence LAMP and PCR reaction system

\begin{tabular}{lll}
\hline UreaseB-1 Primers & Sequence (5'-3') & Length (bp) \\
\hline F3 & AGGAGATAATGATTATATGTCAGGA & 25 \\
B3 & TAACGCTATCACCAGTTGTG & 20 \\
FIP(F1C+F2) & CAACTTGGATAGGACGGTCACCAATTAGTACCAGGAGCAATTAACT & 46 \\
BIP(B1C+B2) & ATTCCATCAGGTACTGCTATTCGTTTCCGTTAACTAAGCCGTT & 44 \\
LOOPF & TCTCTACCTTCGTTCATCACAATT & 24 \\
LOOPB & TTAGTCGGAACACGTGAAGTT & 21 \\
\hline UreaseB-2 Primers & Sequence (5'-3) & Length (bp) \\
\hline F3 & AGGAGATAATGATTATATGTCAGGA & 25 \\
B3 & CACCAGTTGTGATACCATATAGAT & 24 \\
FIP(F1C+F2) & CAACTTGGATAGGACGGTCACCAATTAGTACCAGGAGCAATTAACT \\
BIP(B1C+B2) & ATTCCATCAGGTACTGCTATTCGTTTCCGTTAACTAAGCCGTT \\
LOOPF & TCTCTACCTTCGTTCATCACAATT & 46 \\
LOOPB & TTAGTCGGACACGTGAAGTT & 44 \\
\hline UU PCR primer & Sequence (5'-3) & 24 \\
\hline UU forward & CAGGATCATCAAATCAATTCAC & 21 \\
UU reverse & CATAATGTTCCCCTTCGTCTA & 22 \\
\hline
\end{tabular}

Table 3 Composition of the real-time fluorescence LAMP reaction mix

\begin{tabular}{|c|c|c|}
\hline & Components & Volume \\
\hline Reaction mix & $\begin{array}{l}20 \mathrm{mM} \text { Tris- } \mathrm{HCl}(\mathrm{pH} 8.8) \\
10 \mathrm{mM} \mathrm{KCl} \\
8 \mathrm{mM} \mathrm{MgSO}_{4} \\
10 \mathrm{mM}\left(\mathrm{NH}_{4}\right)_{2} \mathrm{SO}_{4} \\
0.1 \% \text { Tween-20 } \\
1 \mathrm{mM} \text { Betaine } \\
1.6 \mathrm{mM} \text { dNTP }\end{array}$ & $12.5 \mu \mathrm{L}$ \\
\hline Primer mix & $\begin{array}{l}\text { FIP }(1.6 \mu \mathrm{M}) \text { and BIP }(1.6 \mu \mathrm{M}) \\
\text { F3 }(0.2 \mu \mathrm{M}) \text { and B3 }(0.2 \mu \mathrm{M}) \\
\text { FLF }(0.8 \mu \mathrm{M}) \text { and FLB }(0.8 \mu \mathrm{M})\end{array}$ & $1 \mu \mathrm{L}$ \\
\hline Nuclease-free water & & $8 \mu \mathrm{L}$ \\
\hline Bst DNA polymerase & & $1 \mu \mathrm{L}$ \\
\hline SYTO-9 & & $0.5 \mu \mathrm{L}$ \\
\hline DNA template & & $2 \mu \mathrm{L}$ \\
\hline Total volume & & $25 \mu \mathrm{L}$ \\
\hline
\end{tabular}

Reaction condition: $63^{\circ} \mathrm{C}, 45-60 \mathrm{~min}$

the startup of reaction procedure was executed in line with instructions of DNA amplification kit. Meanwhile, negative controls were also performed for each reaction tube. The working solutions were prepared using selected primers. A reaction tube was used for dissolution curve detection and to compare the amplification efficiency of two sets of primers while screening out the highest amplification efficiency without a primer dimer.

\section{Specificity test of real-time fluorescence LAMP}

The genomic DNA of UU standard strain (ATCC 27618) and other common clinical pathogens (including Proteus mirabilis, Klebsiella pneumoniae, Enterococcus aerogenes, Staphylococcus haemolyticus, Pseudomonas aeruginosa, Streptococcus agalactiae, Mycoplasma hominis, Escherichia coli) were extracted and amplified in identical reaction conditions of real-time fluorescence LAMP. The specificity of primers was evaluated.

\section{Sensitivity test of real-time fluorescence LAMP}

The concentration of DNA was quantified with the help of a Thermo Scientific Nanodrop 2000 spectrophotometer after titering with $1 \mu \mathrm{L}$ of TE buffer. The sensitivity of the reaction was assessed using a serial dilution of genomic DNA template, wherein original $2 \mu \mathrm{L}$ of genomic DNA was diluted tenfold with ultrapure water five times. Six concentration gradients were finally attained, which were marked B1, B2, B3, B4, B5, $\mathrm{B} 6$ in order. The real-time fluorescence LAMP reactions with identical total volume and different DNA templates with six concentrations were performed under identical conditions, and the sensitivity of detection was assessed according to amplification curves.

\section{Repeatability test of real-time fluorescence LAMP}

The genomic DNA from one positive bacterial strain and one negative bacterial strain was used for repeatability 
assessment of real-time fluorescence LAMP reaction. The LAMP reactions were performed three times under the same experimental conditions. The same primer used for a repeat test was used to evaluate the reliability of LAMP.

\section{PCR analysis}

PCR detection was amplified in a $25 \mu \mathrm{L}$ volume consisting of $5 \mu \mathrm{L}$ template, $0.5 \mathrm{U}$ of TaKaRa Ex Taq, $0.2 \mathrm{mmol} / \mathrm{L}$ deoxynucleotide mixture, and $10 \times \mathrm{TaKaRa}$ Ex Taq Buffer. For the UU assay, $0.8 \mu \mathrm{mol} / \mathrm{L}$ each of the primers UU forward and UU reverse was used in Table 1 (Kong et al. 2000). Amplification conditions were set with an initial denaturation step for $60 \mathrm{~s}$ followed by 30 cycles for denaturation at $94{ }^{\circ} \mathrm{C}$ for $30 \mathrm{~s}$, primer annealing at $55^{\circ} \mathrm{C}$ for $30 \mathrm{~s}$, and extension at $72{ }^{\circ} \mathrm{C}$ for $60 \mathrm{~s}$ with a final incubation at $72{ }^{\circ} \mathrm{C}$ for $15 \mathrm{~min}$. Thermal profiles were performed on a $\mathrm{C} 1000^{\mathrm{TM}}$ Thermal Cycler (Bio-Rad, Hercules, CA, USA). The Amplified products were analyzed by a microchip electrophoresis system (Bio-Rad, Hercules, CA, USA).

\section{Detection of clinical samples}

Clinical strains were collected from clinical patients who tested positive for UU after solid culture. The secretions were inoculated in UU liquid medium and cultured at $37{ }^{\circ} \mathrm{C}$ for $36-48 \mathrm{~h}$. UU growth when the color of the culture tube changed from yellow to pink, and the liquid clarified. Then the culture medium was filtered and transferred to a solid culture medium for confirmation. UU was positive with the tiny colonies with brown, characteristic $15-50 \mu \mathrm{m}$, under a phase-contrast microscope. Then, the extracted DNA samples isolated from 16 clinical specimens were used as the templates for PCR and LAMP assays.

\section{Statistical analysis}

The counting data were analyzed by Kappa test performed with SPSS 19.0 software (IBM Corporation, Armonk, NY, USA) at 95\% confidence intervals (CI). The clinical sensitivity, specificity, positive predictive values (PPV), and negative predictive values (NPV) of real-time fluorescence LAMP and PCR assays were evaluated using conventional culture as a gold standard method.

\section{Results}

\section{Primer screening test}

Two sets of amplified primers with no-loop structure products showed each reaction curve (Fig. 1).

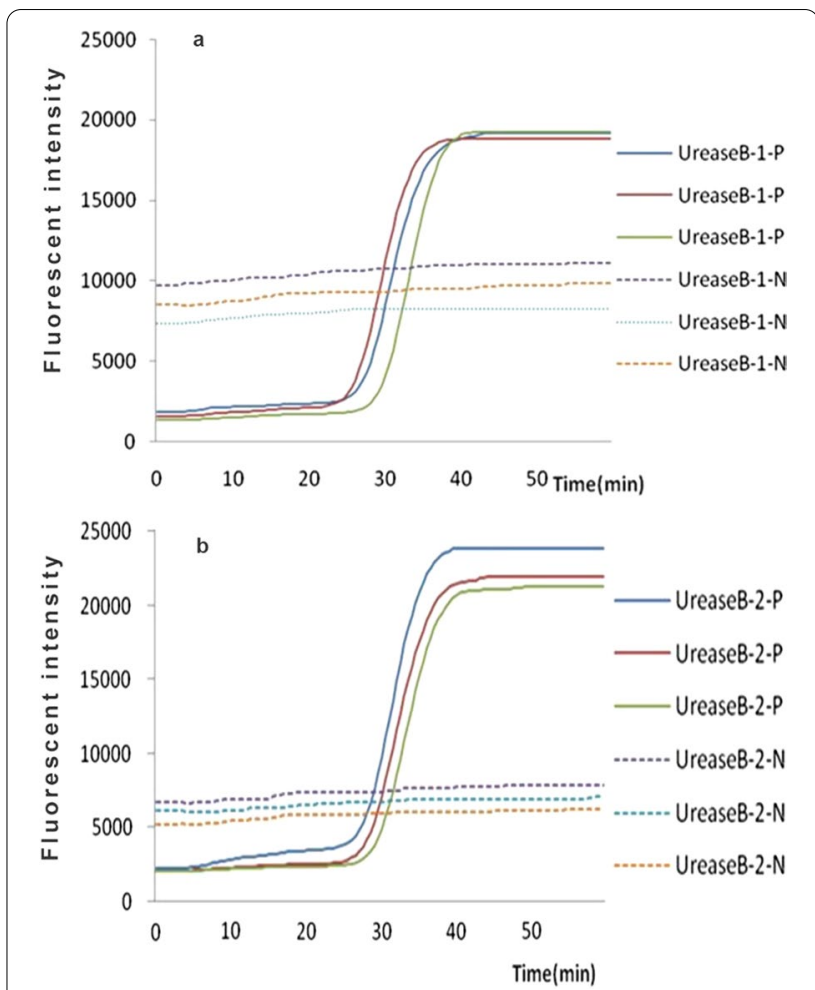

Fig. 1 The primers of real-time fluorescence LAMP screening test

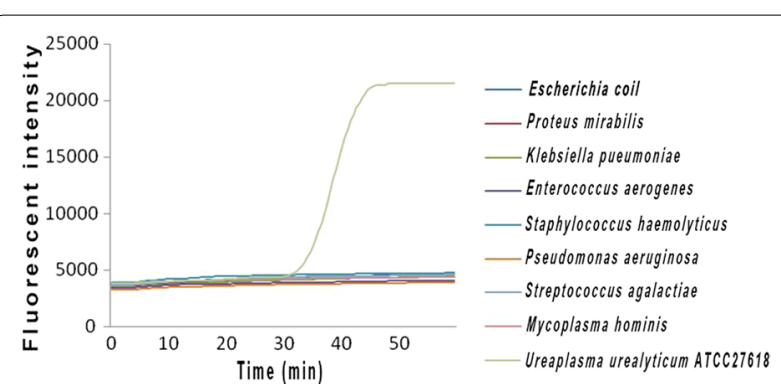

Fig. 2 The specificity of real-time fluorescence LAMP

The visually detectable reaction peak emerged earlier in the reaction with primer UreaseB-2 as compared with primer UreaseB-1, and the fluorescent intensity was also higher in the reaction with primer UreaseB-2 than with primer UreaseB-1 at each check-point. The Urease $B-2$ was the most efficient primer, which had a peak at $40 \mathrm{~min}$ after the initiation of reaction. Also, no amplification was observed in the negative control. Therefore, primer UreaseB-2 had relatively higher amplification efficiency for UU ureaseB gene and was selected for subsequent experiment. 


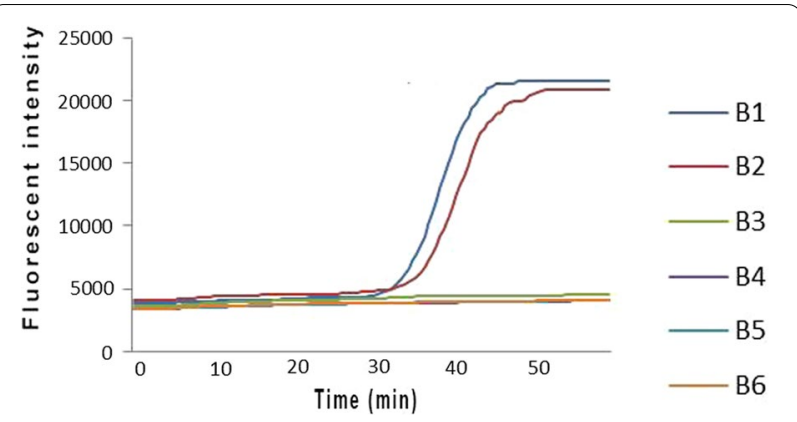

Fig. 3 The sensitivity of real-time fluorescence LAMP

\section{Specificity of real-time fluorescence LAMP}

In the present study, eight common pathogenic bacteria were selected as negative controls and no amplification was tested by real-time fluorescence LAMP assay (Fig. 2). Therefore, the primers for real-time fluorescence LAMP had high specificity, without crossreaction or false-positive results.

\section{Sensitivity of real-time fluorescence LAMP}

The concentration of UU genomic DNA was $420 \mathrm{pg} / \mu \mathrm{L}$, which was diluted by five gradients. Therefore, the resulting concentrations corresponded to 42, 4.2, 0.42, 0.042, $0.0042 \mathrm{pg} / \mu \mathrm{L}$. The sensitivity of UU was $42 \mathrm{pg} / \mu \mathrm{L}$ (Fig. 3). These concentrations correspond to the bacterial concentrations $4.2 \times 10^{6}, 4.2 \times 10^{5}, 4.2 \times 10^{4}, 4.2 \times 10^{3}, 4.2 \times 10^{2}$ and $4.2 \times 10^{1} \mathrm{CFU} / \mathrm{mL}$, respectively. No double-peak appeared on the melting curve with the DNA template at six concentrations, indicating that there was no formation of primer dimers.

\section{Repeatability of real-time fluorescence LAMP}

The reaction peak was visually detected almost simultaneously for the triplicate tubes of a positive sample, and the amplification curves nearly overlapped (Fig. 4). The

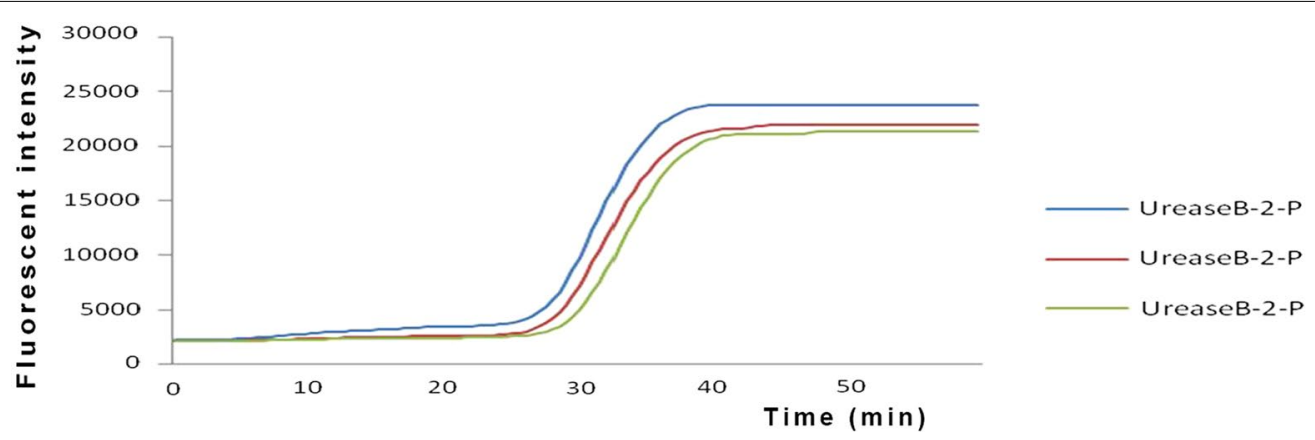

Fig. 4 The repeatability of real-time fluorescence LAMP

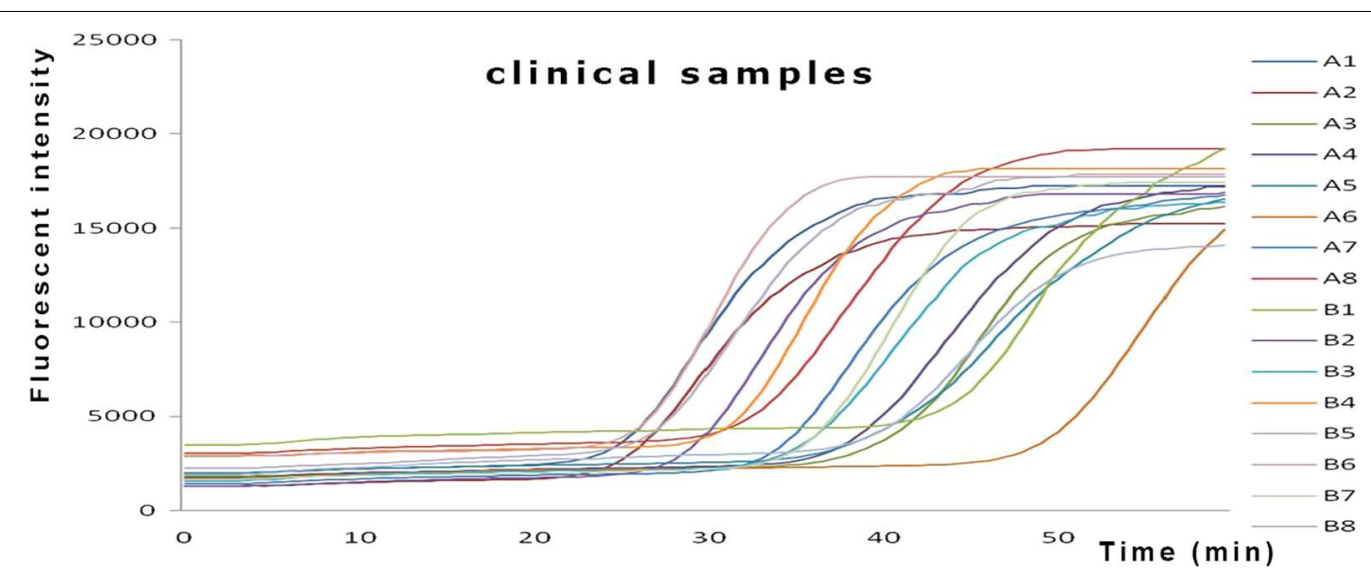

Fig. 5 Detection of common clinical samples by real-time fluorescence LAMP 
Table 4 Comparison of real-time fluorescence LAMP, PCR, and culture results from 16 clinical specimens

\begin{tabular}{|c|c|c|c|c|c|c|c|c|}
\hline \multirow[t]{2}{*}{ Detection of UU } & & \multicolumn{3}{|c|}{ Culture $^{a}$} & \multirow[t]{2}{*}{ Sensitivity $(95 \% \mathrm{Cl})$} & \multirow{2}{*}{$\begin{array}{l}\text { Specificity } \\
(95 \% \mathrm{Cl})\end{array}$} & \multirow[t]{2}{*}{ PPV $(95 \% \mathrm{Cl})$} & \multirow[t]{2}{*}{ NPV $(95 \% \mathrm{Cl})$} \\
\hline & & Pos & Neg & Total & & & & \\
\hline \multirow{2}{*}{$\begin{array}{l}\text { Real-time fluores- } \\
\text { cence LAMP }\end{array}$} & Pos & 16 & 0 & 16 & $100 \%(79.4-100) 16 / 16$ & NA & $100 \%(79.4-100) 16 / 16$ & NA \\
\hline & Neg & 0 & 0 & 0 & & & & \\
\hline \multirow[t]{3}{*}{$P C R^{C}$} & Pos & 14 & 0 & 14 & $87.5 \%(61.7-98.5) 14 / 16$ & NA & $100 \%(76.8-100) 14 / 14$ & NA \\
\hline & Neg & 2 & 0 & 2 & & & & \\
\hline & Total & 16 & 0 & 16 & & & & \\
\hline
\end{tabular}

PPV positive predictive value, NPV negative predictive value

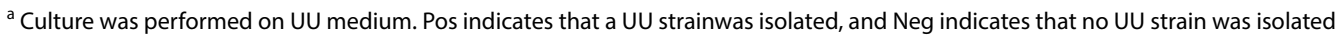

${ }^{b}$ Real-time fluorescence LAMP reaction was confirmed using a real-time quantitative PCR analyzer (ABI 7500). Pos indicates that amplification occurred in the UU LAMP assay, and Neg indicates that amplification did not occur in the UU LAMP assay

${ }^{C} \mathrm{PCR}$ was confirmed using an electrophoretic analysis (C1000 ${ }^{\mathrm{TM}}$ Thermal Cycler). Pos indicates that amplification occurred with the UU PCR assay, and Neg indicates that amplification did not occur in the UU PCR assay

results showed that real-time fluorescence LAMP assay had satisfactory repeatability.

\section{Detection of clinical samples}

Real-time fluorescence LAMP method established in this study and conventional PCR method were used to detect UU in 16 clinical samples simultaneously. Results (Fig. 5) showed that 16 clinical samples were detected positive by LAMP method, while only 14 samples were confirmed to be positive by PCR, showing real-time fluorescence LAMP is superior to PCR in sensitivity.

The overall performances of real-time fluorescence LAMP, conventional PCR, and culture tests used for the detection of UU are shown in Table 4. The sensitivity and PPV of LAMP were 100\% (16/16 specimens; 95\% CI $79.4-100 \%)$ and $100 \%$ (16/16; $95 \%$ CI $79.4-100 \%)$, respectively, compared to culture results. In contrast, the results of conventional PCR were $87.5 \%$ (14/16; 95\% CI $61.7-98.5 \%)$ and $100 \%$ (14/14; 95\% CI 76.8-100.0\%), respectively.

\section{Discussion}

UU is a common human genital tract colonizer, which is frequently related to premature delivery, cesarean section, placental inflammation, fetal lung damage and infant death (Bayraktar et al. 2010). Therefore, rapid detection of UU is of great importance for early diagnose of UU infection.

Conventional diagnosis of UU is based on liquid culture and PCR. Culture is still a gold standard for the detection of UU and provides additional data on antimicrobial sensitivities. However, besides time consumption, conventional culture cannot accurately determine the presence or absence of UU infection, showing there is a certain false-positive rate (Gerber et al. 2003). PCR has been reported to be more sensitive, specific, and rapid than conventional culture method. The clinical sensitivity and specificity of conventional PCR methods have been reported to be from 94 to $95 \%$ and from 91 to $98 \%$, respectively (Abele-Horn et al. 1996; Povlsen et al. 1998). However, PCR-based techniques relied on sophisticated apparatus and complex sample-handling procedures, which limited their application in primary medical facilities. Therefore, given the drawbacks and limitations of conventional detection technologies, an efficient and low-cost method of detecting UU was established in this study based on a real-time fluorescence LAMP assay.

Real-time fluorescence LAMP is a combination of real-time fluorescent technology and isothermal amplification technology with the advantages of being simple, rapid and highly sensitive. LAMP uses Bst DNA polymerase and 4-6 primers that are specific to 4-6 separate regions of target sequences, wherein the reaction is performed in a homothermal condition (Parida et al. 2004). Initially, four primers are used altogether but later, only the inner primers are needed in the cycling reaction. In LAMP reaction, the inner primer hybridizes to target DNA region which gives rise to the formation of a stemloop DNA pattern, and the LAMP cycling reaction starts (Kuboki et al. 2003; Yang et al. 2011). Amplification product shows a characteristic ladder band, and the process is related to the formation of magnesium pyrophosphate (Mori and Notomi 2009). Therefore, LAMP assay allows visualization by naked eye, eliminating the need for cumbersome electrophoresis and ultraviolet observation (Xia et al. 2014), which shows its rapid detection of pathogenic microorganisms. In particular, without the necessity of using expensive thermal circulators, gel electrophoresis and UV detection equipment, LAMP is low-cost. Currently, quantitative LAMP has been developed in broad 
spectrums including cheap semi-quantified E. coli LAMP using paper-based on costly LAMP chips (Rolando et al. 2019; Saengsawang et al. 2021). It is suitable for the rapid detection of a pathogenic microorganism on the spot, the popularization of field and grass-roots in wartime (Mori et al. 2013).

The readout of conventional LAMP mainly relies on white precipitates. However, weak positive results were obtained due to poor amplification efficiency or minute amounts of amplified products (Guo et al. 2018). To overcome this drawback, the fluorescent dye SYTO-9 was used in the LAMP reaction and ABI 7500 system was employed. A real-time fluorescent quantitative PCR instrument was utilized as the LAMP platform, which allowed dynamic and quantitative monitor with increased detection sensitivity. However, SYTO-9 could bind to double-stranded DNA if there was a dimer formation of primers, which could easily lead to false-positive results. To detect potential false-positive results, the melting curve was analyzed and no double-peak was found, indicating no primer dimer formation during the reaction and high specificity of our assay setup. Furthermore, SYTO-9 was added before the reaction and the whole process was completed with closed lid, which can effectively avoid aerosol pollution caused by open-cover detection (Sukphattanaudomchoke et al. 2020).

In the present study, real-time fluorescence LAMP was performed to detect DNA samples for UU standard strain (ATCC 27618) and 8 other strains of common pathogenic bacteria. Positive amplification was only observed in UU, but not in other strains, showing high specificity of LAMP primers and no cross-reactivity with other non-targeted strains. Using conventional culture as a standard, and the sensitivity of the real-time fluorescence LAMP assay reached 100\%, respectively. The high sensitivity of real-time fluorescence LAMP is more impressive when testing with UU standard strain, detecting as low as $42 \mathrm{pg} / \mu \mathrm{L}$ of the DNA concentration, the equivalent of $4.2 \times 10^{5} \mathrm{CFU} / \mathrm{mL}$. This sensitivity was further confirmed with 16 parallel samples of the standard strain. Real-time fluorescence LAMP is carried out to detect UU in 16 clinical samples, while PCR is performed simultaneously. Of all clinical samples tested by PCR, 14 samples were confirmed to be positive while real-time fluorescence LAMP had no missed detection.

However, there are still several limitations in our study. First, given that the number of clinical samples is small in our study, larger and more diverse samples could better determine the sensitivity and reliability of the method. Furthermore, LAMP products are not suitable for expression in cloning and other molecular biology due to various stem loop structures (Mansour et al. 2015). In addition, the specificity of real-time fluorescence LAMP could not be compared with a gold standard test because the specimens used for clinical samples examination were culture-positive.

In conclusion, compared with conventional PCR, real-time fluorescence LAMP for UU detection showed advantages of rapid reaction, high sensitivity, specificity, good repeatability and simple operation, which shows promise of bedside tests and application in primary hospitals.

\section{Abbreviations}

LAMP: Loop-mediated isothermal amplification; UU: Ureaplasma urealyticum.

\section{Acknowledgements}

This research was funded by the Clinical Research Fund of Wu Jieping Medical Genome Association Grant Numbers 320.6750.18455.

\section{Authors' contributions}

JNS, JYY and MXL conceived and designed the study. CQW and DHW participated in data collecting and analysis. JNS, CQW and XYC revised the language of the paper. XGG, WYG and LHL commented on drafts of the paper. All authors read and approved the final manuscript.

\section{Funding}

This work was supported by the Clinical Research Fund of Wu Jieping Medical Genome Association Grant Numbers 320.6750.18455.

\section{Availability of data and materials}

All data generated or analyzed during this study are included in the article.

\section{Declarations}

\section{Ethics approval and consent to participate}

The study was approved by the ethics committees of the participating hospitals and institutes. All procedures performed in the studies involving human participants were by the ethical standards of the institutional and national research committee and with the 1964 Helsinki declaration and its later amendments or comparable ethical standards.

Consent for publication

Not applicable.

\section{Competing interests}

The authors declare that they have no competing interests.

\section{Author details}

${ }^{1}$ Department of Clinical Laboratory Medicine, The Third Affiliated Hospital of Guangzhou Medical University, Guangzhou, China. ${ }^{2}$ Department of Clinical Medicine, The Nanshan School of Guangzhou Medical University, Guangzhou, China. ${ }^{3}$ Department of Clinical Laboratory Medicine, Maternal and Child Health Care Hospital of Guangming District, Shenzhen, China. ${ }^{4}$ Center for Reproduction Medicine, Key Laboratory for Major Obstetric Diseases of Guangdong Province, The Third Clinical School of Guangzhou Medical University, Guangzhou, China. ${ }^{5}$ Department of Blood Transfusion, The Third Affiliated Hospital of Guangzhou Medical University, Guangzhou, China. ${ }^{6}$ Key Laboratory for Major Obstetric Diseases of Guangdong Province, The Third Affiliated Hospital of Guangzhou Medical University, Guangzhou, China. ${ }^{7}$ Key Laboratory of Reproduction and Genetics of Guangdong Higher Education Institutes, The Third Affiliated Hospital of Guangzhou Medical University, Guangzhou, China.

Received: 23 August 2021 Accepted: 29 January 2022 Published online: 11 February 2022 


\section{References}

Abele-Horn M, Wolff C, Dressel P, Zimmermann A, Vahlensieck W, Pfaff F, Ruckdeschel $G$ (1996) Polymerase chain reaction versus culture for detection of Ureaplasma urealyticum and Mycoplasma hominis in the urogenital tract of adults and the respiratory tract of newborns. Eur J Clin Microbio Infect Dis 15:595-598. https://doi.org/10.1007/BF01709369

Bayraktar MR, Ozerol IH, Gucluer N, Celik O (2010) Prevalence and antibiotic susceptibility of Mycoplasma hominis and Ureaplasma urealyticum in pregnant women. Int J Infect Dis 14:e90-e95. https://doi.org/10.1016/j. ijid.2009.03.020

Dehghan Esmatabadi MJ, Bozorgmehr A, Motalebzadeh H, Bodaghabadi N, Farhangi B, Babashah S, Sadeghizadeh M (2015) Techniques for evaluation of LAMP Amplicons and their applications in molecular biology. Asian Pac J Cancer Prev 16:7409-7414

Enomoto Y, Yoshikawa T, Ihira M, Akimoto S, Miyake F, Usui C, Suga S, Suzuki K, Kawana T, Nishiyama Y, Asano Y (2005) Rapid diagnosis of herpes simplex virus infection by a loop-mediated isothermal amplification method. $J$ Clin Microbiol 43:951-955. https://doi.org/10.1128/JCM.43.2.951-955. 2005

Esen B, Gozalan A, Sevindi DF, Demirbas A, Onde U, Erkayran U, Karakoc AE, Hascicek AM, Ergun Y, Adiloglu AK (2017) Ureaplasma urealyticum: presence among sexually transmitted diseases. Jpn J Infect Dis 70:75-79. https://doi.org/10.7883/yoken.JJID.2015.258

Fuwa K, Seki M, Hirata Y, Yanagihara I, Nakura Y, Takano C, Kuroda K, Hayakawa S (2018) Rapid and simple detection of Ureaplasma species from vaginal swab samples using a loop-mediated isothermal amplification method. Am J Reprod Immunol. https://doi.org/10.1111/aji.12771

Gerber S, Vial Y, Hohlfeld P, Witkin SS (2003) Detection of Ureaplasma urealyticum in second-trimester amniotic fluid by polymerase chain reaction correlates with subsequent preterm labor and delivery. J Infect Dis 187:518-521

Guo XG, Zhou YZ, Li Q, Wang W, Wen JZ, Zheng L, Wang Q (2018) Rapid and reliable diagnostic method to detect Zika virus by real-time fluorescence reverse transcription loop-mediated isothermal amplification. AMB Express 8:60. https://doi.org/10.1186/s13568-018-0591-6

Kafetzis DA, Skevaki CL, Skouteri V, Gavrili S, Peppa K, Kostalos C, Petrochilou $\checkmark$, Michalas S (2004) Maternal genital colonization with Ureaplasma urealyticum promotes preterm delivery: association of the respiratory colonization of premature infants with chronic lung disease and increased mortality. Clin Infect Dis 39:1113-1122. https://doi.org/10.1086/424505

Kong F, Ma Z, James G, Gordon S, Gilbert GL (2000) Species identification and subtyping of Ureaplasma parvum and Ureaplasma urealyticum using PCRbased assays. J Clin Microbiol 38:1175-1179

Kuboki N, Inoue N, Sakurai T, Di Cello F, Grab DJ, Suzuki H, Sugimoto C, Igarashi I (2003) Loop-mediated isothermal amplification for detection of African trypanosomes. J Clin Microbiol 41:5517-5524. https://doi.org/10.1128/ JCM.41.12.5517-5524.2003

Kwak DW, Hwang HS, Kwon JY, Park YW, Kim YH (2014) Co-infection with vaginal Ureaplasma urealyticum and Mycoplasma hominis increases adverse pregnancy outcomes in patients with preterm labor or preterm premature rupture of membranes. J Matern Fetal Neonatal Med 27:333-337. https://doi.org/10.3109/14767058.2013.818124

Lee MY, Kim MH, Lee WI, Kang SY, Jeon YL (2016) Prevalence and antibiotic susceptibility of Mycoplasma hominis and Ureaplasma urealyticum in pregnant women. Yonsei Med J 57:1271-1275. https://doi.org/10.3349/ ymj.2016.57.5.1271

Liu ML, Xia Y, Wu XZ, Huang JQ, Guo XG (2017) Loop-mediated isothermal amplification of Neisseria gonorrhoeae porA pseudogene: a rapid and reliable method to detect gonorrhea. AMB Express 7:48. https://doi.org/ 10.1186/s13568-017-0349-6

Mansour SM, Ali H, Chase CC, Cepica A (2015) Loop-mediated isothermal amplification for diagnosis of 18 World Organization for Animal Health (OIE) notifiable viral diseases of ruminants, swine and poultry. Anim Health Res Rev 16:89-106. https://doi.org/10.1017/S1466252315000018

Mori Y, Notomi T (2009) Loop-mediated isothermal amplification (LAMP): a rapid, accurate, and cost-effective diagnostic method for infectious diseases. J Infect Chemother 15:62-69. https://doi.org/10.1007/ s10156-009-0669-9

Mori Y, Kanda H, Notomi T (2013) Loop-mediated isothermal amplification (LAMP): recent progress in research and development. J Infect Chemother 19:404-411. https://doi.org/10.1007/s10156-013-0590-0
Notomi T, Okayama H, Masubuchi H, Yonekawa T, Watanabe K, Amino N, Hase T (2000) Loop-mediated isothermal amplification of DNA. Nucleic Acids Res 28:E63

Parida M, Posadas G, Inoue S, Hasebe F, Morita K (2004) Real-time reverse transcription loop-mediated isothermal amplification for rapid detection of West Nile virus. J Clin Microbiol 42:257-263. https://doi.org/10.1128/ JCM.42.1.257-263.2004

Povlsen K, Jensen JS, Lind I (1998) Detection of Ureaplasma urealyticum by PCR and biovar determination by liquid hybridization. J Clin Microbiol 36:3211-3216. https://doi.org/10.1128/JCM.36.11.3211-3216.1998

Rolando JC, Jue E, Schoepp NG, Ismagilov RF (2019) Real-time, digital LAMP with commercial microfluidic chips reveals the interplay of efficiency, speed, and background amplification as a function of reaction temperature and time. Anal Chem 91:1034-1042. https://doi.org/10.1021/acs. analchem.8b04324

Ruang-Areerate T, Sukphattanaudomchoke C, Thita T, Leelayoova S, Piyaraj P, Mungthin M, Suwannin P, Polpanich D, Tangchaikeeree T, Jangpatarapongsa K, Choowongkomon K, Siripattanapipong S (2021) Development of loop-mediated isothermal amplification (LAMP) assay using SYBR safe and gold-nanoparticle probe for detection of Leishmania in HIV patients. Sci Rep 11:12152. https://doi.org/10.1038/s41598-021-91540-5

Saengsawang N, Ruang-Areerate T, Kesakomol P, Thita T, Mungthin M, Dungchai W (2021) Development of a fluorescent distance-based paper device using loop-mediated isothermal amplification to detect in urine. Analyst 145:8077-8086. https://doi.org/10.1039/d0an01306d

Sobouti B, Fallah S, Mobayen M, Noorbakhsh S, Ghavami Y (2014) Colonization of Mycoplasma hominis and Ureaplasma urealyticum in pregnant women and their transmission to offspring. Iran J Microbiol 6:219-224

Sukphattanaudomchoke C, Siripattanapipong S, Thita T, Leelayoova S, Piyaraj P, Mungthin M, Ruang-Areerate T (2020) Simplified closed tube loop mediated isothermal amplification (LAMP) assay for visual diagnosis of Leishmania infection. Acta Trop 212:105651. https://doi.org/10.1016/j. actatropica.2020.105651

Xia Y, Guo XG, Zhou S (2014) Rapid detection of Streptococcus pneumoniae by real-time fluorescence loop-mediated isothermal amplification. J Thorac Dis 6:1193-1199. https://doi.org/10.3978/j.issn.2072-1439.2014.07.29

Yang B, Wang X, Li H, Li G, Cao Z, Cheng X (2011) Comparison of loopmediated isothermal amplification and real-time PCR for the diagnosis of tuberculous pleurisy. Lett Appl Microbiol 53:525-531. https://doi.org/10. 1111/j.1472-765X.2011.03141.x

\section{Publisher's Note}

Springer Nature remains neutral with regard to jurisdictional claims in published maps and institutional affiliations.

\section{Submit your manuscript to a SpringerOpen ${ }^{\circ}$ journal and benefit from:}

- Convenient online submission

- Rigorous peer review

- Open access: articles freely available online

- High visibility within the field

Retaining the copyright to your article

Submit your next manuscript at springeropen.com 